\title{
Le influenze bizantine nei secoli IX e X tra Campania e Lazio Qualche aspetto
}

\author{
Guglielmo Cavallo
}

Individuare e stabilire quali furono nei secoli IX e X le influenze della civiltà bizantina nell'Italia centro-meridionale in generale, e soprattutto di quale intensità esse furono e in quali modi esse si manifestarono è tema arduo e complesso. Da una parte, infatti, si tratta di un'area nella quale l'eredità grecoromana dell'ultima antichità giocò un peso notevole, e dove perciò non sempre è possibile distinguere tra persistenze locali di antica tradizione e più recenti apporti dall'Oriente bizantino, e d'altra parte, nell'Italia centrale e meridionale territorio peraltro dal variegato quadro geostorico, politico-istituzionale e religioso - la stessa civiltà di Bisanzio non si irradiò da un solo polo, Costantinopoli, ma anche da aree provinciali con le loro specificità, e le influenze furono altresì di indole diversa nel tempo e nei vari ambiti, giungendo per vie e veicoli vari ${ }^{1}$. Va chiarito, altresì, che il concetto di influenza qui non sarà trattato sotto l'aspetto generale dell'Histoire et culture dans l'Italie byzantine $^{2}$, ma sarà inteso nel senso della ricezione della civiltà di Bisanzio, latamente considerata, in contesti fondamentalmente latini e limitatamente alla Campania e al Lazio nei secoli IX e X. Di questa ricezione, peraltro, nei limiti di tempo qui consentiti, non potranno che essere discussi solo alcuni aspetti.

L'impressione più immediata che emerge e che si vuole qui verificare è quella dell'affievolirsi - lungo una linea di demarcazione segnata dal tardo secolo VIII almeno per Roma - di influenze bizantine rispetto al periodo precedente. Nonostante l'elemento greco-orientale fosse rimasto piuttosto numeroso in centri come Napoli e la stessa Roma e si fosse insediato nell'Italia meridionale non solo bizantina, ma anche longobarda, e nonostante vi fosse una continuità di compresenze e incontri etnici tra Greci e Latini e di relazioni con Bisanzio a vari livelli, non sembra tuttavia che nei secoli IX e X la ,grecità abbia

1 Mi limito a rinviare alla lezione spoletina, ormai classica, di Agostino Pertusi: Bisanzio e l'irradiazione della sua civiltà in Occidente nell'alto medioevo, in Centri e vie di irradiazione della civiltà nell'alto medioevo, Spoleto 1964 (Sett. cent. it. 11), pp. 75-133.

2 Il riferimento è al volume: Histoire et culture dans l'Italie byzantine, a cura di André Jacob/Jean-Marie Martin/Ghislaine Noyé, Roma 2006 (Collection de L'École Française de Rome 363), cui si rimanda per le molteplici proiezioni di Bisanzio nell'Italia centrale e meridionale greca. 
giocato un ruolo significativo nella compagine latina in termini di ricezione da parte di quest'ultima.

Si possono prendere le mosse dalla conoscenza della lingua greca e dalle traduzioni dal greco. Ove si eccettuino etnie e individui di stirpe greco-orientale o italo-greca, nei secoli IX e X la conoscenza della lingua greca negli ambienti latini risulta inesistente o assai limitata. $\mathrm{E}$ anche in quest'ultimo caso è da chiedersi: quale conoscenza? A quali livelli? Non è lecito, infatti, parlare per quest'epoca genericamente e semplicemente di ,lingua greca'. Già da lungo tempo - conseguenza della diglossia, vale a dire dell'antica separazione linguistica tra stile attico e koinè - esistevano diverse varietà di greco come mezzo di comunicazione scritta e orale. In tutta l'Italia di cultura latina la conoscenza della lingua greca alta rimase ovviamente del tutto ignorata; né sono testimoniati, del resto, strumenti linguistici adeguati per apprendere la stessa lingua greca. Una ricerca su grammatiche e dizionari greci nell'Europa carolingia ne ritrova l'uso, peraltro modesto, al di là delle $\mathrm{Alpi}^{3}$. Al Lazio, e forse a Roma, sembra potersi attribuire solo il codice di Londra, British Library, Harley 5792, riferibile all'inizio del secolo IX e contenente un glossario greco-latino, quello impropriamente detto "glossario dello pseudo-Cirillo» ${ }^{4}$, ed è di speciale interesse osservare che tracce sicure di questo glossario si trovano comunque tra quel secolo e il successivo nella traduzione in latino, fatta a Roma, del dossier agiografico dei santi edesseni Gurias, Samonas e Abibos 5 . In questa prospettiva di carenza di grammatiche e lessici adeguati sono da valutare perciò certe conoscenze della lingua greca che si dimostrano attestate tra Campania e Lazio.

Si prenda Napoli e la sua cerchia ducale tra la seconda metà del secolo IX e i primi due terzi del X. Dal duca Sergio I ai suoi figli Gregorio III, Atanasio e Stefano, fino a suo nipote Atanasio II e più tardi al lociservator Gregorio e al duca Giovanni III, tutti si possono ritenere - e alcuni sono esplicitamente detti - istruiti nella lingua greca non meno che nella latina ${ }^{6}$. I più commissionano

3 Carlotta Dionisottr: Greek Grammars and Dictionaries in Carolingian Europe, in: The Sacred Nectar of the Greeks: The Study of Greek in the West in the Early Middle Ages, a cura di Michael W. Herren/Shirley Ann Brown, London 1988 (King's College London Mediaeval Studies), pp. 1-56.

4 Si vedano Emma Condello: Una scrittura e un territorio. L'onciale dei secoli V-VIII nell'Italia meridionale, Spoleto 1994 (Biblioteca di Medioevo Latino 12), pp. 100 sg., e Paolo Radiciottri: Manoscritti digrafici grecolatini e latinogreci nell'alto medioevo, in: RHMitt 40 (1998) pp. 91-94.

5 Paolo Chiesa: Il dossier agiografico latino dei santi Gurias, Samonas e Abibos, in: Aevum 65 (1991) pp. 229-232.

6 Mi limito a rimandare a Vera von Falkenhausen: La Campania tra Goti e Bizantini, in: Storia e civiltà della Campania, Il Medioevo, a cura di Giovanni Pugliese Carratelli, Napoli 1992, p. 26, e a Jean-Marie Martin: Hellénisme politique, hellénisme religieux

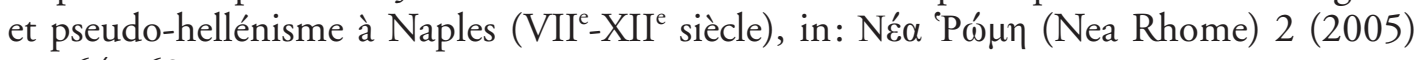
pp. 64-69. 
traduzioni di testi agiografici al circolo di interpreti della cosiddetta „scuola napoletana", e Atanasio II traduce egli stesso la Passio Arethae ${ }^{7}$; ma la lingua greca in cui si dimostrano scritte vite di santi o raccolte di miracoli è solitamente una lingua medio-bassa, talora magari vicina a quella del parlato quotidiano. Queste traduzioni, certo, costituiscono una forma di ricezione della letteratura agiografica bizantina, ma rivelano solo una conoscenza della lingua ad un livello tutt'altro che alto, e in ogni caso si risolvono in una destrutturazione della cultura greca, una sua assimilazione nella cultura dominante, la latina. Né del resto in quest'epoca si trattava sempre di traduzioni vere e proprie, giacché talvolta, in realtà, venivano rielaborate e migliorate traduzioni precedenti, forse del VII e dell'VIII secolo, che risultavano manchevoli o addirittura suscitavano nei lettori o ascoltatori ridiculum potius quam imitationem ${ }^{8}$. Queste riscritture non richiedevano alcuna conoscenza del greco, giacché di sicuro non veniva fatto alcun controllo sui testi greci; ed anzi la vicenda narrata nel greco costituiva più volte „un semplice punto di partenza“ che offriva „il destro al traduttore per una rielaborazione del racconto in direzioni originali“" ( $\mathrm{Si}$ può osservare per inciso, senza peraltro volerne trarre alcuna conseguenza sul piano delle influenze tra Bisanzio e Napoli, che all'incirca nella stessa epoca Simeone Metafrasta a Costantinopoli rielaborava stilisticamente le antiche vite di santi perché scritte in maniera rozza, tanto che, per dirla con Michele Psello, la lettura

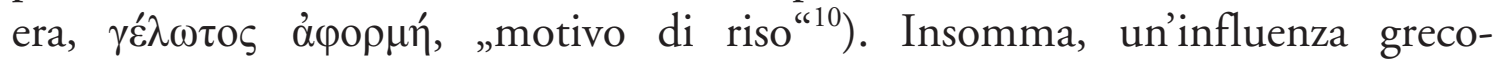
orientale sul santorale napoletano va senz'altro ammessa, come mostra anche il calendario liturgico marmoreo di San Giovanni Maggiore del secolo IX, il quale reca un numero assai alto di santi greci rispetto ai latini ${ }^{11}$, ma questa influenza non implica alcuna autentica conoscenza della lingua greca.

Nell'ambito delle traduzioni dal greco a Napoli va ricordata una testimonianza scarsamente nota anche se ad essa è dedicato un bel contributo di Arsenio Frugoni. La testimonianza conferma ed esplicita il quadro qui tracciato. Si tratta del Prologus della traduzione - eseguita nel secolo X da un arciprete Leone non meglio identificato - del famoso "Romanzo di Alessandro» attribuito allo

7 Si veda soprattutto Paolo Chiesa: Le traduzioni dal greco: l'evoluzione della scuola napoletana nel X secolo, in: Lateinische Kultur im X. Jahrhundert. Akten des I. Internationalen Mittellateinerkongresses (Heidelberg, 12-15.IX.1988), a cura di Walter Berschin, Stuttgart 1991 (=MJb 24/25 [1989-1990]), pp. 67-86; ma riescono utili anche Guglielmo Cavallo: La cultura greca. Itinerari e segni, in: Storia e civiltà della Campania (cit. nota 6), pp. 278-282, e Martin: Hellénisme (cit. nota 6) pp. 64-69.

8 Bonito: «Vita Theodori», in: AASS Febr. 2, Antwerpen 1658, pp. 30sg.

9 Chiesa: Le traduzioni (cit. nota 7) p. 73.

10 Michele Psello: «Encomio per Simeone Metafrasta», in: Michaelis Pselli Orationes hagiographicae, a cura di Elizabeth A. Fischer, Stuttgart/Leipzig 1994, p. 278.

11 Hippolyte Delehaye: Hagiographie napolitaine, in: AnalBoll 57 (1939) pp. 5-64; Domenico Mallardo: Il calendario marmoreo di Napoli, Roma 1947. 
Pseudo-Callistene ${ }^{12}$. In tal Prologus Leone racconta, in terza persona, che egli, dai duchi Giovanni (Giovanni III) e Marino (il giovane figlio di Giovanni) fu inviato con un incarico agli imperatori di Bisanzio Costantino (Costantino VII Porfirogenito) e Romano (Romano II). E qui Leone cepit inquirere libros ad legendum, e tra questi uno ne trovò historiam continentem certamina et victorias Alexandri regis Macedoniae, lo trascrisse e ne portò con se la copia a Napoli rendendone consapevoli i suoi signori. Un giorno il sagacissimus duca Giovanni, ricordando che l'arciprete Leone possedeva il «Romanzo di Alessandro», lo chiamò a sé invitandolo a farne una traduzione de Greco in Latinum. Questo invito ducale si inquadrava in una più vasta attività letteraria e di recupero librario di Giovanni III, il quale, primum, fece raccogliere i libri quos in sua dominatione invenit, nel suo ducato totius Campaniae, e questi renovavit atque meliores effectus (= effecit): il duca dunque volle che tali libri fossero trascritti in nuova veste, ma non prima che vi fossero stati apportati miglioramenti testuali. L'arciprete Leone ci informa anche che si trattava soprattutto di libri sacri. La testimonianza chiarisce molte cose sul modo di procedere dei duchi di Napoli riguardo alle traduzioni: essi verosimilmente recuperavano nel loro ducato totius Campaniae sia modelli greci ivi scritti o giunti in qualche modo, sia libri latini contenenti traduzioni precedenti commissionando, secondo i casi, o traduzioni o rielaborazioni per migliorarne la lingua. Quanto al «Romanzo di Alessandro», testo profano, si trattava in ogni caso di letteratura di consumo, giacché scritto, si sa, in uno stile semplice e colloquiale. La traduzione latina peraltro - più tardi diffusa ampiamente con il titolo «Historia de proeliis» - tradisce la scarsa conoscenza del greco da parte del traduttore ${ }^{13}$, a ulteriore conferma delle competenze linguistiche non più che medio-basse nella Napoli dell'epoca.

Quel che va sottolineato tuttavia è che a Napoli si devono ammettere in quest'epoca pratiche di apprendimento del greco spinte talora - come nel caso dell'entourage ducale e della scuola di traduttori - fino a forme di bilinguismo, magari non perfetto, che peraltro il più delle volte erano limitate alla conoscenza del semplice alfabeto. Si tocca qui un altro fenomeno, spesso ricorrente nelle carte napoletane proprio del IX e del X secolo: la consuetudine da parte di un buon numero di testimoni - un terzo circa del totale attestato - di sottoscrivere gli atti privati in lingua latina ma in caratteri greci ${ }^{14}$. In un caso come quello di

12 L'edizione qui utilizzata è quella di Friedrich Pfister: Der Alexanderroman des Archipresbyter Leo, Heidelberg 1913, pp. 45sg. Sul Prologus si veda Arsenio Frugoni: La biblioteca di Giovanni III duca di Napoli (dal Prologus dell'arciprete Leone al «Romanzo di Alessandro»), in: Annali della Scuola speciale per archivisti e bibliotecari della Università di Roma 9 (1969) pp. 161-171.

13 Chiesa: Le traduzioni (cit. nota 7) p. 82.

14 Sul fenomeno si vedano Francesca Luzzati Laganà: Le firme greche nei documenti del Ducato di Napoli, in: StM, $3^{a}$ ser. 23 (1982) pp. 729-752; Vera von Falkenhausen: A Mediaeval Neapolitan Document, in: The Princeton University Library Chronicle 30 
Napoli, ove il gioco di acculturazioni tra Greci e Latini doveva essere complesso, è difficile risalire dalle firme in caratteri greci alle etnie diverse delle mani agenti. Ma la più parte di quanti sottoscrivevano, a quel che hanno osservato Vera von Falkenhausen e Jean-Marie Martin erano latini, tra cui molti membri della locale gerarchia militare-amministrativa, e non greci; e al di là delle motivazioni sottese al fenomeno - vezzo, emulazione, propensione filo-bizantina, rivendicazione di identità culturale - importa osservare che queste firme indicano solo un'alfabetizzazione greca di base, tanto più ove si trovino adoperate, come sovente, forme maiuscole, le quali mostrano un'istruzione elementare ferma al primo stadio di apprendimento dei segni alfabetici greci, giacché la scrittura minuscola si imparava in una fase successiva. Questi latini, insomma, si

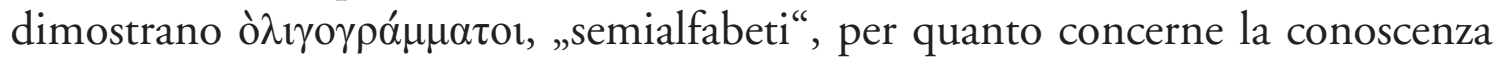
del greco. Può essere di qualche interesse osservare che a Napoli sono testimoniate anche iscrizioni, forse del tardo secolo X, parzialmente in lettere greche ma di lingua latina, quali si conservano su due sarcofagi parietali, posti l'uno sull'altro ed una volta adattati ad altare, nella chiesa di S. Maria a Pugliano ${ }^{15}$.

Da Napoli a Roma. Quanto qui per i secoli IX e X si conoscesse di greco e a quale livello fuori di etnie e insediamenti greci, peraltro perenti a quell'epoca, è difficile dire, ma il vuoto di documentazione - a parte anche qui un certo numero di traduzioni agiografiche dal greco in latino - va interpretato verosimilmente come sostanziale assenza. Si conosce una lettera in greco inviata da papa Pasquale I (817-824) all'imperatore di Bisanzio Leone V (813-820) sul culto delle immagini, ma questa lettera - costruita quasi interamente intessendo citazioni bibliche e qualche ripresa da Giovanni Damasceno dimostra quanto scarsa e pedestre fosse la conoscenza del greco nella cancelleria pontificia $^{16}$. Di lì a poco se ne perderà ogni traccia. Unica luminosa eccezione Anastasio Bibliotecario nella Roma della seconda metà del secolo IX, attivo soprattutto all'epoca dei papi Nicola I, Adriano II e Giovanni VIII ${ }^{17}$. Anastasio traduce testi agiografici, almeno una dozzina di vite di santi, ma anche gli atti

(1968/1969) pp. 171-182, e EAD.: La Campania (cit. nota 6) p. 26; Martin: Hellénisme (cit. nota 6) pp. 73-76.

15 Bartolomeo Capasso: Monumenta ad Neapolitani Ducatus Historiam pertinentia, 2/2, Napoli 1892, pp. 229-232.

16 Thomas F. X. Noble: The Declining Knowledge of Greek in Eight- and Ninth-Century Papal Rome, in: BZ 78 (1985) pp. 56-62.

17 Su Anastasio Bibliotecario e le sue traduzioni dal greco mi limito a rimandare a Girolamo Arnaldi: Anastasio Bibliotecario, in: DBI 3 (1961) pp. 25-37, e a Claudio Leonardi: Anastasio Bibliotecario e l'ottavo concilio ecumenico, in: StM 8 (1967) pp. 59-192, ID.: L'agiografia romana nel secolo IX, in: Hagiographie cultures et sociétés, IV ${ }^{\mathrm{e}}-\mathrm{XII}^{\mathrm{e}}$ siècle. Actes du Colloque organisé à Nanterre et à Paris (2-5 mai 1979), Paris 1981, pp. 471-489, e ID.: Anastasio Bibliotecario e le traduzioni dal greco nella Roma altomedievale, in: The Sacred Nectar (cit. nota 3) pp. 277-296. 
dei concilii ecumenici di Nicea (II, 787) e di Costantinopoli (IV, 869-870), la "Chronographia tripartita di Niceforo», Giorgio Sincello e Teofane Confessore, i cosiddetti «Collectanea», silloge di scritti attinenti al monotelismo quasi tutti dei secoli VII e VIII, e infine alcuni testi teologici. Ma queste traduzioni mostrano solo che a Roma „non si era più in grado, salvo qualche eccezione, di intendere il greco $^{\text {" } 18}$. Le stesse traduzioni di vite di santi, del resto, presentano impianto narrativo, situazioni, quadri mentali e psicologici tante volte lontani da quelli greco-orientali, segno di adattamenti funzionali a esigenze e gusti di chiese locali e di un pubblico diverso ed estraneo rispetto ai destinatari d'origine. Si trattava talora addirittura di retroversioni, come nel caso della passio di s. Dionigi, il cui testo, tradotto da Anastasio e da lui attribuito al patriarca Metodio, altro non era che una versione greca della passio latina composta da Ilduino. È significativa, altresì, l'attenzione dedicata da Anastasio a vite di santi romani quali Acacio e i papi Clemente I e Martino I. In particolare la vita di Martino I è paradigmatica nel mostrare in queste agiografie - ha scritto Claudio Leonardi - „una linea che tende a mettere in rilievo, basandosi sugli stessi testi bizantini, le eresie dei cristiani d'Oriente e i soprusi del potere politico di Costantinopoli, per affermare in ultima istanza, e in opposizione a Bisanzio, l'ortodossia di Roma e la sua autonomia politica “19. È da chiedersi insomma in che limiti si possa parlare di autentiche influenze bizantine, pur ammettendo che attraverso la traduzione di vite di santi l'Italia centro-meridionale fece da intermediaria tra Bisanzio e l'Occidente nel diffondere certe narrazioni di miracoli di immagini. Anastasio Bibliotecario deve essere menzionato anche per la revisione della traduzione fatta da Giovanni Scoto del corpus dello Pseudo-Dionigi l'Areopagita e per la traduzione, che egli stesso fece, degli scholia di Cirillo di Scitopoli e di Massimo il Confessore a tal testo ritrovati in un manoscritto venutogli ad manus nella stessa Roma. Certo, come è stato scritto, dopo la sua scoperta, l',oeuvre du Pseudo-Denys ordonne le monde ${ }^{\text {“20 }}$, ma la sua influenza risulta marcata nella cultura carolingia, mentre meno sembra essersi esercitata nella Roma dei secoli IX e X, nonostante la presenza ne fosse da tempo e saldamente attestata ${ }^{21}$.

18 LeOnARd: Anastasio Bibliotecario e le traduzioni (cit. nota 17) p. 278.

19 ID.: Le traduzioni dal greco nella Roma altomedievale, in: Fatti, patrimoni e uomini intorno all'Abbazia di s. Nilo nel medioevo. Atti del I colloquio internazionale (Grottaferrata, 26-28 Aprile 1985), Grottaferrata 1988, p. 109.

20 Evelyne Patlagean: Les stoudites, l'empereur et Rome: figure byzantine d'un monachisme riformateur, in: Bisanzio, Roma e l'Italia nell'alto medioevo, 1, Spoleto 1988 (Sett. cent. it. 34), p. 434. Si veda anche Jean-Marie SANSTERre: Les moines d'Occident et le monachisme d'Orient du $\mathrm{VI}^{\mathrm{e}}$ au XI $\mathrm{XI}^{\mathrm{e}}$ siècle: entre textes anciens et réalités contemporaines, in: Cristianità d'Occidente e cristianità d'Oriente (secoli VI-XI), 1, Spoleto 2004 (Sett. cent. it. 51), pp. 297sg.

21 Si veda ultimamente Salvatore Lilla: Brief Notes on the Greek Corpus Areopagiticum in Rome during the early middle ages, in: Dionysius 19 (2001) pp. 201-214. 
Si entra, così, in un'altra questione, quella del culto delle immagini, la quale, per quanto qui si dirà, è largamente debitrice ai lavori di Jean-Marie Sansterre ${ }^{22}$. La situazione si presenta fluida e complessa, tanto che lo stesso Sansterre concludeva il suo più ampio intervento sul gioco di influenze ed evoluzioni parallele tra l'Italia e Bisanzio con l'ammissione „ma contribution n'a pas pu répondre à la question “23. Innanzitutto va fatta una distinzione ancora una volta tra VIII e IX secolo: a Roma e più in generale in Italia si fecero sentire i riflessi del primo iconoclasmo, mentre del secondo non si ebbe in sostanza quasi alcuna traccia. Nell'860 Anastasio Bibliotecario, in una lettera all'imperatore Michele III, scritta a nome di papa Nicola I - nel ribattere alle insinuazioni dell'imperatore e del patriarca Fozio circa lo scarso sostegno della Chiesa di Roma al culto delle immagini - costruisce una difesa dottrinale servendosi di materiali del secolo precedente ${ }^{24}$. Ma in ogni caso, ha scritto Sansterre, „les situations romaine et byzantine ne sont pas identiques" ${ }^{\text { } 25}$.

A Roma vi sono, certo, manifestazioni di culto e forme di rappresentazione che richiamano da vicino il funzionamento dell'immagine a Bisanzio: si pensi alla processione dell'Achiropita del Salvatore conservata al Laterano - attestata almeno fino alla metà del secolo IX - la notte precedente la festa dell'Assunta, quando tra un tripudio di lumi, l'immagine, posta accanto ad un'icona della Vergine, veniva esposta alla venerazione di fedeli imploranti ${ }^{26}$, o si pensi ancora, secondo quanto narra Giovanni Immonide verso l'875, all'immagine di Gregorio Magno che era a Sant'Andrea in Clivo Scauri, davanti alla quale la fiammella di un cero talora si accendeva miracolosamente ${ }^{27}$, o si pensi infine all'episodio, sentito a religiosissimis viris e quindi riferito da Liutprando, a detta del quale sanctorum quaedam imagines si inchinarono in San Pietro davanti alle spoglie di papa Formoso - condannato nel processo in presenza del cadavere

22 Jean-Marie SAnsterre: Entre 'koinè méditerranéenne', influences byzantines et particularités locales: le culte des images et ses limites à Rome dans le haut Moyen Âge, in: Europa medievale e mondo bizantino. Contatti effettivi e possibilità di studi comparati. Tavola rotonda del XVIII Congresso del CISH (Montréal, 29 agosto 1995), a cura di Girolamo Arnaldi/Guglielmo Cavallo, Roma 1997 (Nuovi studi storici 40), pp. 109-124, ID.: Attitudes occidentales à l'égad des miracles d'images dans le haut Moyen Âge, in: Annales HSS 53/6 (1998) pp. 1219-1241, e iD.: Entre deux mondes? La vénération des images à Rome et en Italie d'après les textes des $\mathrm{VI}^{\mathrm{e}}-\mathrm{XI}^{\mathrm{e}}$ siècles, in: Roma fra Oriente e Occidente, 2, Spoleto 2002 (Sett. cent. it. 49), pp. 993-1050.

23 SANSTERre: Entre deux mondes? (cit. nota 22) p. 1049.

24 Nicolai I. papae epistolae, ed. Ernst Perels, in: MGH Epp. Karol. 4, Berlin 1925, pp. $436-438$ n. 82.

25 SAnsterre: Entre deux mondes? (cit. nota 22) p. 1006.

26 Michel Andrieu: Les Ordines Romani du Haut Moyen Âge, vol. 5: Les textes (Ordo L), Louvain 1961 (SSL 29), pp. 358-362.

27 Giovanni Diacono: S. Gregori Magni Vita, IV, 85, in: Migne PL 75 col. 231 B. 
dell'897 - per testimoniarne la santità ${ }^{28}$. Né mancano immagini che sanguinano o che cambiano posto nella chiesa cui appartengono. In tutti questi casi di manifestazioni reali o di fatti leggendari è lecito parlare almeno di „une allure byzantine ${ }^{\text {2 } 29}$.

Ma d'altra parte a Roma il culto delle immagini rimase nel complesso un fenomeno più limitato che a Bisanzio, né fu incoraggiato dalla Chiesa, forse perché questa ne temeva una diffusione tale da sfuggire al suo controllo. Vi furono anche altre differenze: la venerazione tributata al crocefisso, che talora opera miracoli - se ne incontra un esempio a Roma nel 921 - non trova riscontro a Bisanzio. E ancora, nonostante nella citata lettera di Nicola I a Michele III si affermi il principio che si debbono venerare le immagini dei santi non meno delle reliquie, in realtà questa equivalenza rimase teorica, giacché a Roma, e più in generale nell'Italia centro-meridionale, le immagini furono relegate in secondo piano rispetto al culto delle reliquie. A Miseno, per esempio, quando nel secolo X si scopre un'immagine musiva di san Sossio, non si esita - a detta del racconto agiografico - a distruggerla sfondando la parete per raggiungere il corpo del santo annunciato dal dipinto ${ }^{30}$. Del resto a Napoli e nell'Italia meridionale un interesse per il culto delle immagini compare non prima del X-XI secolo, forse provocato da immigrazioni di Greci dalla Calabria, mentre prima di quell'epoca manca qualsiasi testimonianza significativa ${ }^{31}$. Ma tra Bisanzio e l'Italia c'è soprattutto una differenza di fondo. A Bisanzio l'immagine ha una giustificazione teologica nel rapporto che lega la figura al prototipo, mentre in Italia, e più in generale in Occidente, questa giustificazione manca, e l'immagine è sostanzialmente ridotta, nel solco del pensiero di Gregorio Magno, a funzione didascalica. In ultima analisi, per quanto concerne il culto delle immagini, la questione delle influenze bizantine tra Campania e Lazio si dimostra, va ribadito, fluida e complessa, e in ogni caso non ne va enfatizzata la portata.

Quando si passi a considerare il monachesimo, non sono da mettere in dubbio né la presenza di monasteri e monaci greci nell'Italia del IX e X secolo né le relazioni che intercorsero, talora anche intense, tra monachesimo greco e monachesimo benedettino, ma che l'orientale lumen avrebbe permeato la

28 Liutprandi Cremonensis Antapodosis, ed. Paolo Chiesa, Turnhout 1998 (CChrCM 156), I, 31, pp. $23 \mathrm{sg}$.

29 SAnsterre: Entre 'koinè méditerranéenne' (cit. n. 22) p. 116.

30 Giovanni Diacono Napoletano: Translatio sancti Sosii, 28, in: AASS Sept. 6, Paris 1867, p. 880 (testo ristampato da Translatio S. Sosii auctore Iohanne Diacono, ed. Georg WAITZ in: MGH SRL, Hannover 1878, pp. 461-462).

31 Jean-Marie Martin: Quelques remarques sur le culte des images en Italie méridionale pendant le haut Moyen Âge, in: Cristianità ed Europa. Miscellanea di studi in onore di Luigi Prosdocimi, a cura di Cesare Alzati, vol. 1/1, Roma/Freiburg/Wien 1994, pp. 223-236. 
magistra latinitas - non solo in Italia ma in tutta l'Europa medievale - fino a penetrare profondamente nella coscienza stessa della cristianità occidentale è tesi di Patricia M. McNulty e Bernard Hamilton che va fortemente ridimensiona$\mathrm{ta}^{32}$. Si prendano proprio i territori longobardo-cassinesi qui considerati, dove nei secoli IX e X coesistevano già da tempo etnie di lingua, tradizioni e rito greco con quelle di lingua e di rito latino, e dove perciò incontro e confronto tra i due monachesimi erano continui e serrati, con presenze di monaci (e monache) greci in monasteri latini e viceversa ${ }^{33}$. Questi contatti culminarono nella visita, storicamente accertata, di san Nilo a Montecassino all'epoca dell'abate Aligerno: narra l'agiografo che „gli venne incontro tutta la comunità dei monaci, anche i sacerdoti e i diaconi, rivestiti degli abiti sacri, come nel giorno festivo, recando in mano ceri e incensieri; e con questa pompa introdussero il beato nel monastero. Sembrava loro di ascoltare e di vedere o il grande Antonio venuto da Alessandria, o meglio, il grande Benedetto, il divino loro legislatore e maestro, resuscitato dai morti “34. Il riferimento ad Antonio, il grande asceta del deserto, indica certo quanto la comunità cassinese rimase colpita e ammirata dalla figura altrettanto ascetica, di san Nilo, dal modello scabro ed eroico di monachesimo antico che egli rappresentava e che era stato a fondamento della istituzione stessa di san Benedetto. Questa ammirazione, infatti, affondava le sue radici in una certa nostalgia dell'antica perfezione, stimolata dalla lettura di traduzioni di vite di santi che si maceravano nel deserto come quella largamente diffusa di santa Maria Egiziaca. Ma l'ammirazione restò soltanto tale, e quel modello di spiritualità non esercitò alcuna influenza sui monaci cassinesi, dediti a

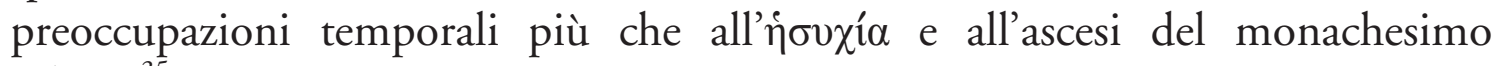
niliano ${ }^{35}$.

32 Patricia M. Mc Nulty/Bernard Hamilton: Orientale lumen et magistra latinitas: Greek Influences on Western Monasticism (900-1100), in: Le Millénaire du Mont Athos. Études et Mélanges, vol. 1, Chevetogne 1963, pp. 181-216, rist. in: Bernard Hamilton: Monastic Reform, Catharism and the Crusades (900-1300), London 1979 (Variorum Reprints. Collected Studies Series 97).

33 Jean-Marie Sansterre: Le monachisme bénédictin et le monachisme italo-grec au $\mathrm{X}^{\mathrm{e}}$ et dans la première moitié du $\mathrm{XI}^{\mathrm{e}}$ siècle: relations et distinctions, in: Il monachesimo italiano dall'età longobarda all'età ottoniana (secc. VIII-X). Atti del Convegno di studi storici sull'Italia benedettina (Nonantola, 10-13 settembre 2003), Cesena 2006, pp. $102-104$.

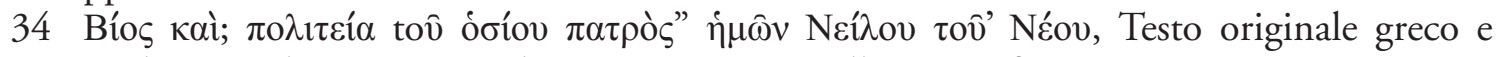
studio introduttivo a cura di Germano Giovanelli, Grottaferrata 1972, p. 112 (cap. 73).

35 Si vedano i lavori di Jean-Marie Sansterre: Saint Nil de Rossano et le monachisme latin, in: Miscellanea di studi in onore di P. Marco Petta, a cura di Augusta Acconcia Longo/Santo Lucì/Lidia Perria, vol. 2, Grottaferrata 1992 (Bollettino della Badia Greca di Grottaferrata n. s. 45 [1991]), pp. 339-386, e iD.: Le monachisme bénédictin (cit. n. 33) pp. 97-118. 
Ma mancarono influenze anche per altri aspetti. L'ubbidienza alla Regola di san Benedetto non ammetteva deroghe ma solo qualche libertà interpretativa ${ }^{36}$ ed era assai lontana dai diversi tipika che monastero per monastero in qualche modo guidavano la vita monastica greca, almeno quando la guidavano, giacché non vanno dimenticate le diffuse forme di idiorritmia. Da un monachesimo greco altalenante tra diversi modelli di vita nella più assoluta libertà e che non conosceva regole di valore generale, nulla potevano recepire le pratiche benedettine. E ancora, la sostanziale stabilità del monachesimo latino ${ }^{37}$ - „il monastero deve essere costruito, se possibile, in modo che ci sia tutto il necessario, ... perché i monaci non siano costretti a girovagare, il che non giova assolutamente alle loro anime“, recita la regola di san Benedetto ${ }^{38}$ - non poteva in alcun modo recepire la tendenza alla forte mobilità o comunque a certe maniere di vita itineranti del monachesimo italo-greco che si dislocava tra Calabria, Lucania, Campania e Lazio. Nessuna influenza, inoltre, si può documentare sotto il profilo culturale: a Montecassino, almeno fino all'inizio del secolo XI, non sono testimoniati monaci di lingua latina che conoscessero il greco, neanche tra i più colti quale un Lorenzo d'Amalfi; né si può provare che vi si trascrissero codici greci; né, ancora, si può documentare alcuna influenza del tipo di codice italo-greco (tecniche librarie, scritture, ordini decorativi) sul tipo di codice cassinese. Manoscritti di opere greche e di scuola niliana, quali l'Ottob. gr. 251 e il Casin. 431, scritti sul tornante tra i secoli X e XI e contenenti letteratura monastica, non furono trascritti nell'abbazia, pur se, a quanto ha dimostrato Enrica Follieri, vi entrarono fin dall'inizio del secolo XI e uno dei due vi si conserva ancora ${ }^{39}$. Ma furono letti da chi? Sembra trattarsi di doni all'abbazia o di acquisizioni al fine di arricchire la biblioteca, ma senza una precisa destinazione a pratiche di lettura. Del resto che solo due codici greci si dimostrino entrati a Montecassino in età antica di fronte alla notevolissima produzione libraria di scuola niliana ${ }^{40}$ è circostanza già di per sé eloquente nel

36 SAnsterre: Le monachisme bénédictin (cit. nota 33) pp. 99sg.

37 Si veda quanto scrive Jean-Marie SANSTERre: Attitudes à l'égard de l'errance monastique en Occident du VI ${ }^{\mathrm{e}}$ au XI siècle, in: Voyages et voyagerurs à Byzance et en Occident du $\mathrm{VI}^{\mathrm{e}}$ au XI $\mathrm{XI}^{\mathrm{e}}$ siècle. Actes du Colloque international organisé par la Section d'Histoire de l'Université libre de Bruxelles en collaboration avec le Departement des Sciences Historiques de l'Université de Liège (5-7 mai 1994), a cura di Alain Dekkers/JeanMarie Sansterre/Jean-Louis Kupper, Genève 2000, pp. 215-234.

38 La règle de saint Benoît, ed. Adalbert de VogüÉ/Jean Neufville, Paris 1972 (SC 182), 66, 6-7, p. 660.

39 Enrica Follieri: Due codici greci già cassinesi oggi alla Biblioteca Vaticana: gli Ottob. gr. 250 e 251, in: Palaeographica, diplomatica et archivistica. Studi in onore di Giulio Battelli, vol. 1, Roma 1979 (Storia e letteratura 139), pp. 159-221.

40 Si vedano almeno i lavori di Santo Lucà: Attività scrittoria e culturale a Rossano: da s. Nilo a s. Bartolomeo da Simeri (secoli X-XII), in: Atti del Congresso internazionale su s. Nilo di Rossano (28 settembre-1 ottobre 1986), Rossano/Grottaferrata 1989, 
testimoniare un sostanziale disinteresse per la cultura greca. Il monachesimo benedettino ignorò del tutto o quasi persino testi greci fondamentali per la formazione del monaco, quali il «Prato spirituale» di Giovanni Mosco, la «Scala del Paradiso» di Giovanni Climaco, le «Catechesi» di Teodoro Studita. Insomma il soggiorno di san Nilo a Montecassino e la sua successiva permanenza insieme al suo seguito nel monastero di San Michele a Vallelucio, una dipendenza cassinese, e più in generale i molti contatti tra i due monachesimi in tutta l'area longobardo-cassinese, nella compagine del monachesimo latino non ebbero che una portata assai limitata su qualsiasi piano: della spiritualità, delle pratiche devozionali, della cultura, degli schemi mentali e dei comportamenti concreti inerenti alla professione monastica. Vi furono solo alcuni individui di quella compagine (il futuro abate di Montecassino Giovanni III, il monaco Liutius) che si sentirono spinti a vivere esperienze della spiritualità greco-orientale soggiornando in luoghi come la Terra Santa, il Sinai, l'Athos ${ }^{41}$.

Qualche cenno sul monachesimo anche a Napoli e a Roma. A Napoli non sembra che nei secoli IX e X monaci e monasteri greci abbiano esercitato una qualche influenza spirituale e culturale sui monaci latini, limitandosi questi a sottoscrivere talora in caratteri greci, più volte peraltro in lingua latina e rozzamente, o a imparare, magari attraverso il solo ascolto, rudimenti del canto liturgico greco. Anzi, molti monasteri non conservavano di greco che solo il nome ${ }^{42}$. Quanto a Roma, si suole invocare il monastero dei Santi Bonifacio e Alessio sull'Aventino - fondato nel 977 da Sergio, metropolita di Damasco fattosi monaco - come „crocevia dell'incontro fra Oriente greco e Occidente latino, fra cristianità mediterranea e cristianità europea" che assolveva "la funzione di ponte fra le diverse culture e anime della chiesa e di centro donde si irradiavano esperienze e modelli culturali e spirituali da un limite all'altro dell'orbe cristiano “43. Ma se è vero che il monastero dei Santi Bonifacio e Alessio godette di visite di Graeci optimi ${ }^{44}$ e di relazioni privilegiate con l'Oriente ${ }^{45}$,

pp. 25-73, e ID.: Scritture e libri della scuola niliana, in: Scritture, libri e testi nelle aree provinciali di Bisanzio. Atti del Seminario di Erice (18-25 settembre 1988), a cura di Guglielmo Cavallo/Giuseppe De Gregorio/Marilena Maniaci, vol. 1, Spoleto 1991 (Biblioteca del Centro per il collegamento degli studi medievali e umanistici nell'Università di Perugia 5), pp. 319-387.

41 Vera von Falkenhausen: Il monachesimo italo-greco e i suoi rapporti con il monachesimo benedettino, in: L'esperienza monastica benedettina e la Puglia. Atti del Convegno di studio organizzato in occasione del XV centenario della nascita di san Benedetto (Bari/Noci/Lecce/Picciano, 6-10 ottobre 1980), a cura di Cosimo Damiano Fonseca, Galatina 1983, pp. 128sg.; Sansterre, Le monachisme bénédictin (cit. nota 33) p. 111.

42 Su tutta la questione si veda Martin: Hellénisme (cit. nota 6) pp. 69-73.

43 Filippo Burgarella: Presenze greche a Roma: aspetti culturali e religiosi, in: Roma fra Oriente e Occidente (cit. nota 22) p. 986. 
giacché ad esso si rivolse Oreste patriarca di Gerusalemme per chiedere disposizioni ecclesiali, sembra comunque doversi escludere che all'interno del monastero convivesse una comunità greca accanto alla latina, ciascuna con la sua osservanza; e d'altra parte è argomento di discussione se il monastero dei Santi Bonifacio e Alessio abbia veramente esercitato una vasta influenza sul monachesimo occidentale trasmettendo modi e pratiche della spiritualità greco-orientale ${ }^{46}$. Anche in questo caso si trattò piuttosto di scelte individuali: si pensi ad Adalberto di Praga, che in quel monastero si umiliò fino a svolgere $\mathrm{i}$ lavori più servili come gli antichi asceti ${ }^{47}$.

E vengo alle conclusioni. Nei secoli IX e X mancano significative influenze di Bisanzio nell'Italia centro-meridionale latina. La stessa Roma si stacca man mano dall'Oriente, con cui non ha più relazioni politiche o religiose di qualche peso. Nonostante presenze greche a diversi livelli e nei diversi quadri della società - presenze talora forti ed estese dalla Sicilia a Roma - e nonostante una produzione di testi, di libri, di documenti, di iscrizioni, di monete, di prodotti artistici greci, non vi fu da parte dei Latini una ricezione della lingua, della cultura, della spiritualità greca più che superficiale o individuale. Alle influenze non certo marcate, che si sono man mano rilevate non è molto quel che si può aggiungere: istituzioni caritatevoli come le diaconie, ispirate a Napoli e a Roma da modelli greco-orientali ${ }^{48}$, o certi usi documentari come la continuità nell'uso del papiro come supporto sempre a Roma, a Napoli e in qualche altro ambito italo-meridionale ${ }^{49}$, o stilemi cancellereschi di ascendenza bizantino-imperiale che si son voluti vedere nella scrittura curiale romana antica della cancelleria pontificia $^{50}$, o a Roma sintomi greci nella scrittura e nella decorazione dei fogli di restauro 55-70 del Vat. lat. 3836, il cosiddetto omiliario di Agimondo, o il tracciato secondo maniere greche della minuscola carolina del codice Düsseldorf

44 Jean-Marie SAnsterre: Le monastère des Saints-Boniface et Alexis sur l'Aventin et l'expansion du christianisme dans le cadre de la "Renovatio Imperii Romanorum» d'Otton III, in: RevBén 100 (1990) pp. 502sg., e ID.: Saint Nil de Rossano (cit. nota 35) pp. 373-380.

45 Burgarella (cit. nota 43) p. 987.

46 Su questi argomenti si veda sempre Sansterre: Saint Nil de Rossano (cit. nota 35) pp. 379sg. e 385sg., e ID.: Le monachisme bénédictin (cit. nota 33) pp. 107-111.

47 Mc Nulty/Hamilton (cit. nota 32) p. 211.

48 Per Roma si veda il classico lavoro di Henry-Irénée Marrou: L'origine orientale des diaconies romaines, in: MAH 57 (1940) pp.95-142, e per Napoli Martin: Hellénisme (cit. nota 6) pp. 60-62.

49 ID: Chartula in tumbo scripta, bolumen chartacium. Le papyrus dans les duchés tyrrhéniens pendant le haut Moyen Âge, in: MEFRM 112 (2000) pp. 183-189.

50 Si veda almeno Jan-Olof T JäDER: Le origini della scrittura curiale romana, in: Bullettino dell'Archivio Paleografico Italiano, $3^{a}$ ser. $2-3$ (1963/1964) pp. 7-54. 
E 1 dell'epoca di Giovanni VIII, o altro del genere ${ }^{51}$. Resta, certo, sullo sfondo, il problema delle influenze bizantine su arte e programmi iconografici di quell'epoca: problema assai indagato e dibattuto. Ultimamente, a questo proposito, i mosaici per la chiesa di San Marco a Roma, commissionati da Gregorio IV (827-844) sono stati considerati, tra l'altro, come difesa della validità delle immagini contro l'iconoclasmo ${ }^{52}$. Ma in fatto di influenze storicoartistiche è da chiedersi più in generale con Hagen Keller: „ist die Rezeption

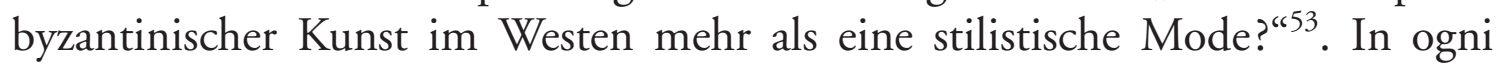
caso argomenti relativi alle arti figurative o agli assetti urbanistici, così come temi di carattere politico, economico, militare, non rientrano nel mio discorso, limitato come questo è ad alcuni aspetti delle influenze bizantine.

Lingua, maniere del sociale, usi, testi e pratiche devozionali, sottigliezze teologiche, ideali monastici, arti, forme grafiche della grecità esercitarono un loro fascino o acquisirono un qualche prestigio nella compagine latina; e sono questo fascino, questo prestigio che spiegano certi fenomeni di cosiddetta ,ellenizzazione', i quali tuttavia non servirono a promuovere né una più esperta conoscenza della lingua greca né una coscienza e un'assunzione dei valori della grecità nell'Italia centro-meridionale. Bisanzio è destrutturata nelle traduzioni in latino, è ridotta a modesto segno grafico nelle sottoscrizioni, è ripresa come civiltà delle immagini svuotata di significati, è proposta come modello spirituale senza alcuna convinta adesione.

Senza nascondersi la complessità e le distinzioni inerenti al problema dell',alterità' tra Greci e Latini, fascino della grecità e vocazioni ,ellenizzanti' si dimostrano per lo più di facciata. La realtà sono invece una diffidenza e un'avversione profonde verso Bisanzio. Anastasio Bibliotecario taccia i Greci di difetti connaturali come la furberia e l'inaffidabilità ${ }^{54}$. In un contesto di contatti continui e intensi come quello tra Campania e Lazio, la cronachistica latinocassinese non è aliena da termini come „superbia“ e "tracotanza“ rivolti ai Greci $^{55}$. Su questa diffidenza, su questa avversione dei Latini nei confronti dei Greci (che trovano del resto riscontro in atteggiamenti analoghi di Bisanzio

51 Guglielmo Cavallo: Interazione tra scrittura greca e scrittura latina a Roma tra VIII e IX secolo, in: Miscellanea codicologica François Masai dicata, a cura di Pierre Cockshaw/Monique-Cécile Garand/Pierre Jodogne, Gand 1979, pp. 23-29; John Osborne: The Use of Painted Initials by Greek and Latin Scriptoria in Carolingian Rome, in: Gesta 29 (1990) pp. 76-85.

52 Claudia Bolgia: The Mosaics of Gregory IV at S. Marco, Rome: Papal Response to Venice, Byzantium and the Carolingians, in: Speculum 81 (2006) pp. 1-34.

53 Hagen Keller: Das ottonische Kirchenreich und Byzanz, in: Cristianità d'Occidente (cit. nota 20) p. 254.

54 Arnaldi (cit. nota 17) p. 26.

55 Chronica Monasterii Casinensis, ed. Hartmut Hoffmann, Hannover 1980 (MGH SS 34), II, 37, p. 237. 
verso il mondo latino) ${ }^{56}$ il discorso si potrebbe allargare, oltre i confini dei territori qui considerati, all'Italia in generale e magari all'Europa, ma sarebbe discorso lungo ${ }^{57}$. Mi sia consentito ricordare solo due testimonianze del secolo $\mathrm{X}^{58}$. Mendax Graecia esclama Raterio di Verona citando Giovenale (Sat. X 174), l'autore antico che si scagliava contro qualsiasi alterità etnica ${ }^{59}$. Ma è soprattutto la lettura di Liutprando di Cremona che si rivela assai istruttiva: Liutprando nella sua prosa $\mathrm{fa}$ dotto sfoggio di termini greci che egli ha imparato a padroneggiare nel corso delle sue ambascerie e dei suoi soggiorni in Oriente e di cui sente il fascino ${ }^{60}$; ma nella sua considerazione i Greci sono „gente ignobile“, „uomini molli, effeminati, menzogneri, di nessun sesso, poltroni“, da loro "promanano tutte le eresie" o tutte le "ciance vuote", il loro basileus veste e mangia in modi indecenti ed è mendace, superbo, avido ${ }^{61}$. E dunque, al di là dell'ammirazione, della superficiale imitazione o delle occasionali incidenze, nella compagine latina emerge un sostanziale rigetto antropologico di Bisanzio.

\section{Zusammenfassung}

Obwohl im 9. und 10. Jahrhundert Einzelne und Gruppen griechisch-orientalischer Herkunft sich sowohl im byzantinischen und auch langobardischen Süditalien als auch in Zentralitalien niederließen, und obwohl sie relativ zahlreich in Zentren wie Neapel und Rom waren, scheint es dennoch nicht so, als ob die byzantinischen Einflüsse auf die lateinische Basis signifikant und ausgeprägt gewesen wären. Die Kenntnis der griechischen Sprache in den lateinischen Milieus erweisen sich als nicht bestehend oder sehr gering: die Übersetzung der Heiligenviten und einiger anderer Texte vom Griechischen ins

56 SAnStERre: Le monachisme bénédictin (cit. n. 33) p. 107.

57 Si vedano almeno il classico Herbert Hunger: Graeculus perfidus - 'I $\tau \alpha \lambda$ òs i $\tau \alpha \mu$ ós. Il senso dell'alterità nei raporti greco-romani ed italo-bizantini, con una introduzione di Otto Kresten, Roma 1987, e ultimamente Chris Wickнam: Ninth Century Byzantium through Western Eyes, in Byzantium in the Ninth Century: Dead or Alive? Papers from the Thirtieth Spring Symposium of Byzantine Studies, Birmingham, March 1996, a cura di Leslie Brubaker, Aldershot/Brookfield/Singapore/Sidney 1998 (Society for the Promotion of Byzantine Studies. Publications, 5), pp. 245-256.

58 Una raccolta di testimonianze di quest'epoca con discussione critica si deve a Michael Rentschler: Griechische Kultur und Byzanz im Urteil westlicher Autoren des 10. Jahrhunderts, in: Saeculum 29 (1978) pp. 324-355.

59 Die Briefe des Bischofs Rather von Verona, ed. Fritz Weigle, Weimar 1949 (MGH Epp. DK 1), p. 30 n. 5.

60 Johannes KODER: Liutprand von Cremona und die griechische Sprache, in: ID./Thomas Weber: Liutprand von Cremona in Konstantinopel, Wien 1980, pp. 17-61.

61 Liutprandi Cremonensis Relatio de legatione Constantinopolitana, 9, 22, 28, 40, 54, ed. Chiesa (cit. nota 28) pp. 191, 196 sg., 199, 204 sg, 211. 
Lateinische in Neapel offenbaren eine spärliche Kenntnis der Originalsprache oder aber Bearbeitungen von lateinischen Übersetzungen, die in der vorhergehenden Epoche gemacht worden sind. In Rom kann richtige Kenntnis der griechischen Sprache allein Anasthasius Bibliothecarius zuerkannt werden; er war als Übersetzer vieler griechischer Texte zur Zeit der Päpste Nikolaus I., Hadrian II. und Johannes VIII. tätig. In Rom blieb auch der Bilderkult - ein Phänomen von großer Bedeutung in den byzantinischen Kultpraktiken beschränkt und wurde vom Papsttum nicht gefördert, vielleicht weil befürchtet wurde, dass eine große Verbreitung stattfinden und die Kirche die Kontrolle darüber verlieren könne. Das Mönchtum betreffend, gibt es sichere Beweise sowohl für die Präsenz von griechischen Klöstern und Mönchen als auch für Beziehungen zwischen dem griechischen und benediktinischen Mönchtum, jedoch fehlen bedeutsame Einflüsse der griechisch-orientalischen Spiritualität auf lateinische Mönche, mit einigen, einzelnen Ausnahmen. Byzanz und seine Kultur übten einen gewissen Einfluss auf das lateinische Element aus, aber diese Faszination war nur eine Äußerliche, während in Wirklichkeit Teile der Lateiner den Griechen Misstrauen und Abneigung entgegenbrachten. Diese verhinderten ein wirkliches Eindringen der byzantinischen Einflüsse zwischen Kampanien und Latium in dieser Epoche. 
Bereitgestellt von | De Gruyter / TCS

Angemeldet | 217.89.40.26

Heruntergeladen am | 21.12.12 10:48 